PROCEEDINGS OF THE

AMERICAN MATHEMATICAL SOCIETY

Volume 129, Number 3, Pages 913-919

S 0002-9939(00)05553-2

Article electronically published on September 19, 2000

\title{
LE DEGRÉ DE LINDELÖF EST l-INVARIANT
}

\author{
AHMED BOUZIAD
}

(Communicated by Alan Dow)

\begin{abstract}
Two Tychonoff spaces $X$ and $Y$ are said to be $l$-equivalent if $C_{p}(X)$ and $C_{p}(Y)$ are linearly homeomorphic. It is shown that if $X$ and $Y$ are $l$-equivalent, then the Lindelöf numbers of $X$ and $Y$ are the same. The proof given is a strengthening of the one given by N.V. Velichko to show that the Lindelöf property is $l$-invariant.
\end{abstract}

\section{INTRODUCTION}

Deux espaces topologiques complétement réguliers $X$ et $Y$ sont dits $t$-équivalents (respectivement, l-équivalents) si $C_{p}(X)$ et $C_{p}(Y)$ sont homéomorphes (respectivement, linéairement homéomorphes). Ici $C_{p}(X)$ désigne l'ensemble des fonctions $f: X \rightarrow \mathbb{R}$ continues, muni de la topologie de la convergence simple. L'objet de cet article est de montrer que deux espaces $l$-équivalents quelconques ont le même degré de Lindelöf, ce qui répond à une question de A.V. Arhangelskiǐ reprise dans [4. Les premières suspicions à l'égard de l'invariance du degré de Lindelöf par l-équivalence, ont commencé dès la parution du travail de A.V. Arhangel'skiĩ [1] suivi de celui de E.G. Pytkeev 9], où il est montré, entre autres, que le maximum des degrés de Lindelöf des puissances finies d'un espace est un $t$-invariant. Depuis, plusieurs résultats se rapportant à cette question ont été établis. Un survol complet de l'historique des $t$-invariants et des $l$-invariants se trouve dans les articles 3], [4] de A.V. Arhangel'skiǐ, par conséquent nous en indiquons seulement quelques éléments. Parmi ces résultats, citons O.G. Okunev [7] qui a montré que la $\sigma$-compacité est un $t$-invariant et V.V. Tkachuk [10] qui a montré que le degré héréditaire de Lindelöf est un $l$-invariant. Ce résultat de Tkachuk a été ensuite généralisé par O.G. Okunev 8] en montrant que le degré héréditaire de Lindelöf est en fait un $t$-invariant. Une importante persée dans cette direction, comme l'a dit Arhangel'skiǐ dans [3], a été accomplie en 1990 par N.V. Velichko qui a montré que la classe des espaces de Lindelöf est stable par $l$-équivalence. Ce résultat de N.V. Velichko est paru récemment dans $[12$.

D'autres résultats concernant l'invariance du degré de Lindelöf par l-équivalence, dans des classes particulières d'espaces topologiques, sont connus. D'abord, il résulte immédiatement du résultat de Tkachuk cité ci-dessus que, dans la classe des espaces parfaits, le degré de Lindelöf est un l-invariant (et plus généralement,

Received by the editors January 20, 1999 and, in revised form, May 14, 1999.

2000 Mathematics Subject Classification. Primary 54C35; Secondary 46E10.

Key words and phrases. Set-valued maps, Lindelöf degree, linear homeomorphism, function spaces. 
un $t$-invariant d'après Okunev [8]). J. Baars [5] a montré que c'est également le cas pour la classe des espaces qui sont paracompacts et qui vérifient le premier axiome de dénombrabilité. Le résultat de J. Baars a été étendu par V. Valov [11] à la classe des $w q$-espaces et à celle des espaces $\mu$-complets.

Pour finir cette introduction, nous donnons une brève description de cette note. Le noyau de notre approche est la notion de $\phi$-extracteur introduite et étudiée sous une forme abstraite dans la section 1. Étant donnée une multifonction $\phi: X \rightarrow$ $\mathcal{P}(Y)$ semi-continue inférieurement, un $\phi$-extracteur est une application $G$ définie sur la collection des ouverts de $Y$ et à valeurs dans les parties de $X$ et reliée à $\phi$ par des propriétés spécifiques. (La définition précise est donnée ci-dessous.) Une telle application $G$, quand elle existe, est connectée à $\phi$ de telle sorte que, sous des conditions convenables, le degré de Lindelöf $l(Y)$ de $Y$ ne peut pas dépasser celui de $X$ (Proposition 3 et Théorème 4). Dans la section 2, nous considérons un plongement linéaire $\psi: C_{p}(Y) \rightarrow C_{p}(X)$ et nous montrons, en empruntant notamment des idées à [12, que la multifonction $\phi: X \rightarrow \mathcal{P}(Y)$ naturellement associée à $\psi$ admet un $\phi$-extracteur (Lemme 5). Le reste de la preuve (Lemme 6 et 7) consiste à montrer que ce $\phi$-extracteur satisfait à toutes les conditions requises pour que les résultats de la section 1 s'appliquent. Le résultat le plus général (Théorème 8) obtenu par cette méthode s'énonce de la façon suivante: si la multifonction associée au plongement linéaire $\psi: C_{p}(Y) \rightarrow C_{p}(X)$ est à valeurs non vides, alors $l(Y) \leq l(X)$. En particulier, si $X$ et $Y$ sont $l$-équivalents, alors $l(X)=l(Y)$.

\section{Multifonctions et Degré De LindelöF}

Soit $\phi: X \rightarrow \mathcal{P}(Y)$ une multifonction, où $X$ et $Y$ sont deux espaces topologiques et où $\mathcal{P}(Y)$ désigne l'ensemble de toutes les parties de $Y$. L'espace $Y$ est supposé de Hausdorff. Pour tout $A \subset Y$, on note $\phi^{*}(A)=\{x \in X: \phi(x) \subset A\}$ et $\phi(X)=\bigcup\{\phi(x): x \in X\}$. On dira que $\phi$ est surjective si $Y=\phi(X)$.

Tout au long de cette note, les lettres $\mathbb{N}$ et $\mathbb{R}$ désignent respectivement l'ensemble des entiers positifs non nuls et l'espace usuel des nombres réels. Le symbole $|A|$ désigne le cardinal de l'ensemble $A$.

Rappelons que $\phi: X \rightarrow \mathcal{P}(Y)$ est dite semi-continue inférieurement si pour tout ouvert $U \subset Y$, l'ensemble $\{x \in X: \phi(x) \cap U \neq \emptyset\}$ est un ouvert de $X$. L'espace $Y$ étant de Hausdorff, le résultat suivant est immédiat.

Proposition 0. Si $\phi$ est semi-continue inférieurement, alors, pour tout $n \in \mathbb{N}$ et pour tout ouvert $V \subset Y$, l'ensemble $\{x \in X:|\phi(x) \cap V| \geq n\}$ est un ouvert de $X$.

Désignons par $\mathcal{T}$ la collection des ouverts de $Y$. On appelle $\phi$-extracteur (ou simplement extracteur) toute application $G: \mathcal{T} \rightarrow \mathcal{P}(X)$ vérifiant les conditions suivantes.

$\mathrm{S}(1): \phi^{*}(U) \subset G(U)$;

S(2): Si $U \subset V$ et $x \in G(V) \backslash G(U)$ alors $\phi(x) \cap(V \backslash U) \neq \emptyset$;

$\mathrm{S}(3)$ : Pour toute suite croissante $\left(U_{n}\right)_{n \in \mathbb{N}} \subset \mathcal{T}$ telle que $X \subset \bigcup_{n \in \mathbb{N}} \bigcap_{m \geq n} G\left(U_{m}\right)$, on a $Y \subset \bigcup_{n \in \mathbb{N}} U_{n}$.

Pour tout $U \in \mathcal{T}$, on note $F(U)=X \backslash G(U)$.

Dans les lemmes 1, 2 et la proposition $3, G$ est un $\phi$-extracteur fixé.

Lemme 1. Soit $\left(U_{n}\right)_{n \in \mathbb{N}} \subset \mathcal{T}$ une suite croissante et soit $x \in X$. Si $\phi(x)$ est fini, alors l'ensemble $I=\left\{n \in \mathbb{N}: x \in G\left(U_{n}\right)\right\}$ est fini ou co-fini. 
Preuve. Supposons que $I$ et $I^{c}$ soient infinis. Alors il existe une suite $\left(n_{k}\right)_{k \in \mathbb{N}} \subset I^{c}$ telle que $n_{k+1} \geq n_{k}+1$ et $n_{k}+1 \in I$ pour tout $k \in \mathbb{N}$. En particulier, d'après $\mathrm{S}(2)$, pour tout $k \in \mathbb{N}$, on a $\phi(x) \cap\left(U_{n_{k}+1} \backslash U_{n_{k}}\right) \neq \emptyset$. Comme les ensembles $U_{n_{k}+1} \backslash U_{n_{k}}$, $k \in \mathbb{N}$, sont deux à deux disjoints, il en résulte que $\phi(x)$ est infini.

Dans le lemme suivant $\left(U_{n}\right)_{n \in \mathbb{N}}$ désigne une suite croissante d'éléments de $\mathcal{T}$ telle que $|\phi(x)| \geq n-k$ pour tous $x \in \bigcap_{i=k}^{n} F\left(U_{i}\right)$ et $k \leq n$.

Lemme 2. Si $\phi(x)$ est fini, alors il existe un entier $n$ tel que $x \in G\left(U_{m}\right)$ pour tout $m \geq n$.

Preuve. D'après le lemme 1, il suffit de montrer que l'ensemble $I=\{n \in \mathbb{N}: x \in$ $\left.G\left(U_{n}\right)\right\}$ est infini. Si $I$ était fini, il existerait $k \in \mathbb{N}$ tel que $x \in \bigcap_{i=k}^{n} F\left(U_{i}\right)$ pour tout $n \geq k$. On aurait alors $|\phi(x)| \geq n-k$ pour tout $n \in \mathbb{N}$, ce qui est impossible puisque $\phi(x)$ est fini.

Le résultat suivant nécessite quelques notations (utilisées également dans la section 2). Pour toute collection $\mathcal{L}$ de sous-espaces de $X$, on note $l(\mathcal{L})=\sup \{l(Z)$ : $\left.Z \in \mathcal{L}_{f}\right\}$, où $l(Z)$ désigne le degré de Lindelöf de $Z$ et $\mathcal{L}_{f}$ la collection de toutes les intersections finies d'éléments de $\mathcal{L}$. Rappelons que le degré de Lindelöf d'un espace topologique $Z$ est le plus petit cardinal infini $\tau$ tel que de tout recouvrement ouvert de $Z$ on puisse extraire un sous-recouvrement de cardinal inférieur ou égal à $\tau$.

Soit $\tau$ un cardinal infini. Pour toute collection $\mathcal{U}$ d'ensembles, on note $[\mathcal{U}]_{\tau}$ la collection constituée des réunions de toutes les sous-collections de $\mathcal{U}$ ayant un cardinal inférieur ou égal à $\tau$. Un recouvrement $\mathcal{U}$ de $Y$ sera dit $\tau$-trivial si $Y \in[\mathcal{U}]_{\tau}$; i.e. $\mathcal{U}$ admet un sous-recouvrement ayant un cardinal inférieur ou égal à $\tau$.

Désignons par $\mathbf{H}(\tau)$ l'énoncé suivant.

"Il existe une collection $\mathcal{L} \subset \mathcal{P}(X)$ vérifiant $l(\mathcal{L}) \leq \tau$ et une base $\mathcal{B}$ de $Y$ telle que, pour tout recouvrement ouvert $\mathcal{U} \subset \mathcal{B}$ de $Y$ non $\tau$ trivial, chaque $U \in[\mathcal{U}]_{\tau}$ soit contenu dans au moins un $V \in[\mathcal{U}]_{\tau}$ tel que $F(V) \in \mathcal{L}$."

Si l'énoncé $\mathbf{H}(l(X))$ est satisfait, on dira que l'extracteur $G$ est synchronisé avec le degré de Lindelöf de $X$.

Proposition 3. Supposons que $\phi$ soit semi-continue inférieurement et à valeurs finies non vides. Si $\mathbf{H}(\tau)$ est satisfait et $l(X) \leq \tau$, alors $l(Y) \leq \tau$.

Preave. Nous supposons que $l(Y)>\tau$ pour aboutir à une contradiction. Fixons $\mathcal{L}$ et $\mathcal{B}$ dont l'existence est guarantie par le fait que $\mathbf{H}(\tau)$ est vrai. Fixons un recouvrement ouvert $\mathcal{U} \subset \mathcal{B}$ de $Y$ non $\tau$-trivial. Nous allons construire, par récurrence sur $n$, une suite $\left(U_{n}\right)_{n \in \mathbb{N}} \subset[\mathcal{U}]_{\tau}$ croissante telle que, en posant $F_{k, m}=\bigcap_{i=k}^{m} F\left(U_{i}\right)$ pour $k \leq m$, on ait la propriété $\mathcal{P}_{n}$ suivante

(1) $F\left(U_{i}\right) \in \mathcal{L}$ pour tout $i \leq n$;

(2) $|\phi(x)| \geq m-k+2$ pour tous $x \in F_{k, m}$ et $k \leq m \leq n$;

(3) $\left|\phi(x) \cap U_{m+1}\right| \geq m-k+2$ pour tous $x \in F_{k, m}$ et $k \leq m<n$.

Comme $l(X) \leq \tau$, il existe une collection $\mathcal{U}_{1} \subset \mathcal{U}$ ayant un cardinal inférieur ou égal à $\tau$ telle que $\phi(x) \cap \bigcup \mathcal{U}_{1} \neq \emptyset$ pour tout $x \in X$. Soit $U_{1} \in[\mathcal{U}]_{\tau}$ tel que l'on ait à la fois $\bigcup \mathcal{U}_{1} \subset U_{1}$ et $F\left(U_{1}\right) \subset \mathcal{L}$. Comme $\phi^{*}\left(U_{1}\right) \subset G\left(U_{1}\right)$ et $x \in \phi^{*}\left(U_{1}\right)$ pour tout $x \in X$ tel que $|\phi(x)|=1$, on a $|\phi(x)| \geq 2$ pour tout $x \in F\left(U_{1}\right)$. Supposons que pour $n \in \mathbb{N}$, les ensembles $U_{1} \subset \ldots \subset U_{n}$ vérifiant les conditions (1)-(3) aient 
été définis. Comme $l(\mathcal{L}) \leq \tau$, d'après (1) dans $\mathcal{P}_{n}$ on a $l\left(F_{k, n}\right) \leq \tau$ pour tout $k \leq n$. Donc, compte tenu de la Proposition 0 et de la condition (2) dans $\mathcal{P}_{n}$, il existe une collection $\mathcal{U}_{n+1} \subset \mathcal{U}$ ayant un cardinal inférieur ou égal à $\tau$ telle que $\left|\phi(x) \cap \bigcup \mathcal{U}_{n+1}\right| \geq n-k+2$ pour tout $x \in F_{k, n}$ et pour tout $k \leq n$. Soit $U_{n+1} \in[\mathcal{U}]_{\tau}$ tel que l'on ait à la fois $U_{n} \cup \cup \mathcal{U}_{n+1} \subset U_{n+1}$ et $F\left(U_{n+1}\right) \in \mathcal{L}$. Puisque $l(\mathcal{L}) \leq \tau$, les conditions (1) et (3) de $\mathcal{P}_{n+1}$ sont vérifiées. Montrons que (2) l'est aussi. Nous avons seulement à examiner le cas de $F_{k, n+1}$. Soit $x \in F_{k, n+1}$.

Si $k=n+1$, alors, puisque $\left|\phi(x) \cap U_{1}\right| \geq 1$ et $U_{1} \subset U_{n+1}$, on a $\left|\phi(x) \cap U_{n+1}\right| \geq 1$. Comme $x \in F\left(U_{n+1}\right)$, il résulte de $\mathrm{S}(1)$ que $|\phi(x)| \geq 2=(n+1)-(n+1)+2$.

Si $k<n+1$, alors $F_{k, n+1} \subset F_{k, n}$, donc $x \in F_{k, n}$. Par construction de $U_{n+1}$, on a $\left|\phi(x) \cap U_{n+1}\right| \geq n-k+2$. Comme $x \in F\left(U_{n+1}\right)$, il résulte de $\mathrm{S}(1)$ que $|\phi(x)| \geq n-k+2+1$.

Ceci montre que $\mathcal{P}_{n+1}$ est entièrement vérifiée et termine la construction.

D'après la condition (2), le lemme 2 s'applique à la suite $\left(U_{n}\right)_{n \in \mathbb{N}}$, donc $X \subset$ $\bigcup_{n \in \mathbb{N}} \bigcap_{m>n} G\left(U_{m}\right)$. Par conséquent, d'après $\mathrm{S}(3)$, on a $Y \subset \bigcup_{n \in \mathbb{N}} U_{n}$. Comme $U_{n} \in[\mathcal{U}]_{\tau}$ pour tout $n \in \mathbb{N}$, le recouvrement $\mathcal{U}$ de $Y$ est $\tau$-trivial, ce qui est contradictoire. Donc $l(Y) \leq \tau$.

La proposition 3 entraine le résultat suivant.

Théorème 4. Si $\phi$ est à valeurs finies non vides et admet un extracteur synchronisé avec le degré de Lindelöf de $X$, alors $l(Y) \leq l(X)$.

\section{Application}

Dans cette section, nous appliquons le théorème 4 de la section 1 pour établir le résultat annoncé dans le titre de cette note. Dans toute la suite, $X$ et $Y$ sont deux espaces complétement réguliers et $\psi: C_{p}(Y) \rightarrow C_{p}(X)$ est une application linéaire continue fixés. Rappelons d'abord quelques notions et propriétés de base prevenant de la $C_{p}$-théorie (on pourra consulter [2], 6], 8] pour les démonstrations). On associe de façon standard à $\psi$ une multifonction semi-continue inférieurement $\phi: X \rightarrow \mathcal{P}(Y)$ de la façon suivante. Pour tout $x \in X$, la forme linéaire $\psi_{x}: f \in$ $C_{p}(Y) \rightarrow \psi(f)(x) \in \mathbb{R}$ appartient à $L(Y)$, le dual topologique de $C_{p}(Y)$. Comme les fonctions d'évaluations $f \in C_{p}(Y) \rightarrow f(y) \in \mathbb{R}, y \in Y$, forment une base de Hamel pour $L(Y)$, si $\psi_{x} \neq 0$ il existe un ensemble fini $\phi(x) \subset Y$ non vide et des nombres réels non nuls $\lambda_{y}(x), y \in \phi(x)$, uniques, tels que pour tout $f \in C(Y)$ on ait

$$
\psi(f)(x)=\sum_{y \in \phi(x)} \lambda_{y}(x) f(y) .
$$

Si $\psi_{x}=0$, on pose $\phi(x)=\emptyset$ et on adopte la convention $\sum \emptyset=0$. Ainsi, la multifonction $\phi$ est à valeurs non vides si et seulement si $\psi_{x} \neq 0$ pour tout $x \in X$.

Rappelons que si $\psi$ est un plongement de $C_{p}(Y)$ dans $C_{p}(X)$, c'est-à-dire si $\psi: C_{p}(Y) \rightarrow \psi(C(Y)) \subset C_{p}(X)$ est un homémorphisme, alors $\phi$ est surjective.

Dans la suite, pour simplifer les notations, on posera $\psi(f)=\bar{f}$. En particulier, l'ensemble

$$
\left\{x^{\prime} \in X:\left|\bar{f}\left(x^{\prime}\right)-\bar{f}(x)\right|<1\right\}
$$

est un voisinage ouvert de $x$ dans $X$.

Maintenant nous sommes prêt pour définir un extracteur pour la multifonction $\phi$. Pour $V \subset Y$ et $x \in X$, on note $r_{V}(x)=\sum\left\{\lambda_{y}(x): y \in \phi(x) \cap V^{c}\right\}$ et on pose 
$G(V)=\left\{x \in X: r_{V}(x)=0\right\}$. Le symbole $r_{V}$ est emprunté à [12, où les points de $G(V)$ sont dits $V$-spécifiques.

Lemme 5. Si $\psi: C_{p}(Y) \rightarrow C_{p}(X)$ est un plongement, alors $G$ est un $\phi$-extracteur.

Preuve. Il est clair que $\phi^{*}(V) \subset G(V)$. D'autre part, pour tout $x \in G(V) \backslash G(U)$, avec $U \subset V$, il résulte de l'égalité

$$
r_{U}(x)=r_{V}(x)+\sum_{y \in \phi(x) \cap(V \backslash U)} \lambda_{y}(x) \neq 0
$$

et du fait que $r_{V}(x)=0$, que $\phi(x) \cap(V \backslash U) \neq \emptyset$. Les conditions $\mathrm{S}(1)$ et $\mathrm{S}(2)$ sont donc vérifiées par $G$ (définie sur la collection $\mathcal{T}$ de tous les ouverts de $Y$ ).

Vérifions la condition $\mathrm{S}(3)$. Soit $(U)_{n \in \mathbb{N}} \subset \mathcal{T}$ une suite croissante telle que $X \subset \bigcup_{n \in \mathbb{N}} \bigcap_{m \geq n} G\left(U_{m}\right)$; posons $U=\bigcup_{n \in \mathbb{N}} U_{n}$ et supposons qu'il existe $y \in Y \backslash U$. Posons $O=\{g \in C(Y):|g(y)|<1\}$; comme $\psi: C_{p}(Y) \rightarrow \psi(C(Y))$ est ouverte, il existe $x_{1}, \ldots, x_{l} \in X$ tels que $g \in \psi(O)$ pour tout $g \in \psi(C(Y))$ vérifiant $g\left(x_{i}\right)=0$ pour tout $1 \leq i \leq l$. Posons $F=\bigcup_{i \leq l} \phi\left(x_{i}\right)$ et fixons $k \in \mathbb{N}$ tel que l'on ait à la fois $F \cap U \subset U_{k}$ et $\left\{x_{1}, \ldots, x_{l}\right\} \subset G\left(U_{k}\right)$. Enfin, soit $f \in C(Y)$ telle que $f(F \cap U) \subset\{0\}$ et $f\left(\left(F \cap U^{c}\right) \cup\{y\}\right) \subset\{1\}$. Pour tout $1 \leq i \leq l$, on a

$$
\bar{f}\left(x_{i}\right)=\sum_{z \in \phi\left(x_{i}\right) \cap U} \lambda_{z}\left(x_{i}\right) f(z)+\sum_{z \in \phi\left(x_{i}\right) \cap U^{c}} \lambda_{z}\left(x_{i}\right) f(z)=r_{U}\left(x_{i}\right)=r_{U_{k}}\left(x_{i}\right)=0 .
$$

Donc $\bar{f} \in \psi(O)$. Comme $\psi$ est injective, on obtient $|f(y)|<1$ ce qui est contradictoire. Donc $Y \subset U$.

Désignons par $\mathcal{B}$ la collection des ouverts fonctionnels de $Y$ et $\operatorname{par} \mathcal{C}$ la collection de leurs complémentaires. Rappelons qu'un ouvert $V \subset Y$ est dit fonctionnel s'il existe une fonction continue $f: Y \rightarrow \mathbb{R}$ et un ouvert $U \subset \mathbb{R}$ tels que $V=f^{-1}(U)$. Tout ouvert fonctionnel $V$ admet une décomposition de la forme $V=\bigcup_{n \in \mathbb{N}} F_{n}$ où $F_{n} \in \mathcal{C}$ et $F_{n} \subset F_{n+1}$ pour tout $n \in \mathbb{N}$. Si de plus on peut trouver une telle décomposition vérifiant $\phi^{*}(V) \backslash \phi^{*}\left(F_{n}\right) \neq \emptyset$ pour tout $n \in \mathbb{N}$, on dira que $V$ est adéquat.

Un ensemble $A \subset X$ est dit de type $F_{\tau}$ dans $X$, où $\tau$ est un cardinal, si $A$ s'écrit comme réunion d'une collection ayant un cardinal inférieur ou égal à $\tau$ et constituée de fermés de $X$.

Lemme 6. Soit $S$ un ensemble infini et $\left(V_{s}\right)_{s \in S}$ une famille d'ouverts adéquats, stable pour la réunion finie. Alors $F\left(\bigcup_{s \in S} V_{s}\right)$ est de type $F_{\tau}$ dans $X$, où $\tau=|S|$.

Preuve. Posons $V=\bigcup_{s \in S} V_{s}$. Pour tout $s \in S$, soit $\left(F_{n}^{s}\right)_{n \in \mathbb{N}}$ une décomposition de $V_{s}$ et, pour tout $n \geq 1$, soit $f_{n}^{s} \in C(Y)$ telle que $f_{n}^{s}(x)=0$ si $x \in F_{n}^{s}$ et $f_{n}^{s}(x)=n$ si $x \notin V_{s}$. Pour $x \in \phi^{*}\left(V_{s}\right)$ et $k \in \mathbb{N}$, désignons par $U_{k}^{s}(x)$ l'ensemble

$$
\bigcap_{i \leq k}\left\{x^{\prime} \in X:\left|\bar{f}_{i+n_{x}^{s}}^{s}\left(x^{\prime}\right)\right|<1\right\},
$$

où $n_{x}^{s}$ est le premier entier $m$ tel que $\phi(x) \subset F_{m}^{s}$. Alors, puisque $\bar{f}_{i+n_{x}^{s}}^{s}(x)=0$, $U_{k}^{s}(x)$ est un ouvert de $X$ contenant le point $x$. Posons

$$
A_{s}=\bigcap_{k \in \mathbb{N}} \bigcup_{x \in \phi^{*}\left(V_{s}\right)} U_{k}^{s}(x)
$$


et

$$
B_{s}=\left\{x \in X: \phi(x) \cap\left(V \backslash V_{s}\right) \neq \emptyset\right\}
$$

et soit $A=\bigcap_{s \in S}\left(A_{s} \cup B_{s}\right)$.

L'ensemble $A_{s}$ est un $G_{\delta}$ de $X$. L'ensemble $B_{s}$ est aussi un $G_{\delta}$ de $X$; en effet, comme $\phi$ est à valeurs finies, on a $B_{s}=\bigcap_{n \in \mathbb{N}}\left\{x \in X: \phi(x) \cap\left(V \backslash F_{n}^{s}\right) \neq \emptyset\right\}$. Par conséquent, pour établir le lemme, il suffit de montrer que $G(V)=A$.

Montrons que $F(V) \subset X \backslash A$. Soit $y \in F(V)$. Comme $\phi(y)$ est fini et la famille $\left(V_{s}\right)_{s \in S}$ est stable pour la réunion finie, il existe $s \in S$ tel que $\phi(y) \cap V \subset V_{s}$, i.e. $y \notin B_{s}$. Soit $k \in \mathbb{N}$ tel que l'on ait $\phi(y) \cap V \subset F_{k}^{s}$. Comme $r_{V}(y) \neq 0$, on suppose sans perte de généralité que $k\left|r_{V}(y)\right| \geq 1$. Nous allons vérifier que

$$
y \notin \bigcup_{x \in \phi^{*}\left(V_{s}\right)} U_{k}^{s}(x)
$$

il en résultera que $y \notin A_{s}$ et donc $y \notin A$. Soit $x \in \phi^{*}\left(V_{s}\right)$; on a $\bar{f}_{k+n_{x}^{s}}^{s}(y)=$ $\left(k+n_{x}^{s}\right) r_{V}(y)$, il résulte donc des inégalités $\left(k+n_{x}^{s}\right)\left|r_{V}(y)\right| \geq k\left|r_{V}(y)\right| \geq 1$ que $y \notin U_{k}^{s}(x)$.

Montrons que $X \backslash A \subset F(V)$. Comme les $V_{s}, s \in S$, sont adéquats, on peut supposer que la décomposition de chaque $V_{s}$ est telle que $\phi^{*}\left(V_{s}\right) \backslash \phi^{*}\left(F_{n}^{s}\right) \neq \emptyset$ pour tout $n \in \mathbb{N}$. Soit $y \notin A$ et soit $s \in S$ tel que $y \notin A_{s} \cup B_{s}$. On a $\phi(y) \cap V \subset V_{s}$; soit $p \in \mathbb{N}$ un entier tel que $\phi(y) \cap V \subset F_{p}^{s}$. Fixons $k \in \mathbb{N}$ tel que $y \notin \bigcup_{x \in \phi^{*}\left(V_{s}\right)} U_{k}^{s}(x)$, et $x \in \phi^{*}\left(V_{s}\right)$ tel que $\phi(x) \cap\left(F_{p}^{s}\right)^{c} \neq \emptyset$; on a alors $n_{x}^{s}>p$ et il existe $i \leq k$ tel que $\left|\bar{f}_{i+n_{x}^{s}}^{s}(y)\right| \geq 1$. On a

$$
\phi(y) \cap V=\phi(y) \cap V_{s} \subset F_{p}^{s} \subset F_{i+n_{x}^{s}}^{s},
$$

donc $\bar{f}_{i+n_{x}^{s}}^{s}(y)=\left(i+n_{x}^{s}\right) r_{V}(y)$. Par conséquent, $\left(i+n_{x}^{s}\right)\left|r_{V}(y)\right| \geq 1$; en particulier $r_{V}(y) \neq 0$, donc $y \in F(V)$.

Lemme 7. Supposons que $\phi$ soit surjective. Soit $\tau$ un cardinal infini et $\mathcal{U} \subset \mathcal{B}$ un recouvrement ouvert de $Y$ non $\tau$-trivial. Alors, pour toute famille $\left(V_{s}\right)_{s \in S} \subset \mathcal{U}$, où $|S| \leq \tau$, il existe une famille $\left(U_{t}\right)_{t \in T} \subset[\mathcal{U}]_{\aleph_{0}}$ stable pour la réunion finie telle que

(1) $|T| \leq \tau$;

(2) pour tout $t \in T, U_{t}$ est adéquat;

(3) $\bigcup_{s \in S} V_{s} \subset \bigcup_{t \in T} U_{t}$.

Preuve. Posons $V=\bigcup_{s \in S} V_{s}$ et notons que $[\mathcal{U}]_{\aleph_{0}} \subset \mathcal{B}$. Comme $\mathcal{U}$ est non $\tau$ trivial, on a $Y \backslash V \neq \emptyset$; soit $x_{1} \in X$ tel que $\phi\left(x_{1}\right) \not \subset V$ et $U_{1} \in[\mathcal{U}]_{\aleph_{0}}$ tel que $\phi\left(x_{1}\right) \subset U_{1}$. Supposons que $x_{1}, \ldots, x_{n}$ et $U_{1}, \ldots, U_{n}$ soient construits. L'ensemble $Y \backslash\left(V \cup U_{1} \cup \ldots \cup U_{n}\right)$ est non vide, donc il existe $x_{n+1} \in X$ tel que $\phi\left(x_{n+1}\right) \not \subset V \cup$ $U_{1} \cup \ldots \cup U_{n}$. Soit $U_{n+1} \in[\mathcal{U}]_{\aleph_{0}}$ tel que $\phi\left(x_{n+1}\right) \subset U_{n+1}$. Désignons par $\left(x_{n}\right)_{n \in \mathbb{N}} \subset$ $X$ et $\left(U_{n}\right)_{n \in \mathbb{N}} \subset[\mathcal{U}]_{\aleph_{0}}$ les suites obtenues en poursuivant ce processus. Posons $U=\bigcup_{n \in \mathbb{N}} U_{n}$. Soit $\left(W_{t}\right)_{t \in T}$ la famille de toutes les réunions finies d'éléments de $\left(V_{s}\right)_{s \in S}$ et pour $t \in T$ posons $U_{t}=W_{t} \cup U$. Il est clair que la famille $\left(U_{t}\right)_{t \in T} \subset[\mathcal{U}]_{\aleph_{0}}$ est stable pour la réunion finie, et que l'on a $|T| \leq \tau$ et $\bigcup_{s \in S} V_{s} \subset \bigcup_{t \in T} U_{t}$. Vérifions que chaque $U_{t}$ est adéquat. Soit $t \in T$ et fixons une décomposition $\left(F_{n}^{t}\right)_{n \in \mathbb{N}}$ pour $W_{t}$ et pour chaque $n \in \mathbb{N}$ une décomposition $\left(F_{k}^{n}\right)_{k \in \mathbb{N}}$ pour $U_{n}$. La suite $\left(G_{n}\right)_{n \in \mathbb{N}}$, où $G_{n}=F_{n}^{t} \cup F_{n}^{1} \cup \ldots \cup F_{n}^{n}$, est une décomposition de $U_{t}$; de plus, on a $\left(x_{n}\right)_{n \in \mathbb{N}} \subset \phi^{*}\left(U_{t}\right)$ et $x_{n+1} \notin \phi^{*}\left(G_{n}\right)$ pour tout $n \in \mathbb{N}$.

Nous sommes maintenant en mesure d'établir les résultats annoncés. 
Théorème 8. Supposons que $\psi: C_{p}(Y) \rightarrow C_{p}(X)$ soit un plongement linéaire et que pour tout $x \in X$ il existe $f \in C(Y)$ telle que $\psi(f)(x) \neq 0$. Alors $l(Y) \leq l(X)$.

Preuve. L'hypothèse faite sur $\psi$ assure que la multifonction $\phi$ est à valeurs non vides. D'après le lemme $5, G$ est un $\phi$-extracteur. De plus, d'après les lemmes 6 et 7, l'énoncé $\mathbf{H}(l(X))$ est satisfait lorsque $\mathcal{L}$ est la collection des ensembles qui sont de type $F_{l(X)}$ dans $X$ et $\mathcal{B}$ est la base de $Y$ constituée des ouverts fonctionnels (notons que $\mathcal{L}$ est stable pour l'intersection finie et que $l(\mathcal{L}) \leq l(X)$ ). En d'autres termes, $G$ est un $\phi$-extracteur synchronisé avec $l(X)$. Donc, d'après le théorème 4 , on a $l(Y) \leq l(X)$.

Corollaire 9. Si $X$ et $Y$ sont l-équivalents, alors $l(X)=l(Y)$.

\section{REFERENCES}

[1] A.V. Arhangel'skiǐ, On some topological spaces occurring in functional analysis, Uspehi Mat. Nauk 31, N 5 (1976), 17-32. (In Russian). MR 56:16569

[2] A.V. Arhangel'skiř, Topological Function Spaces, (Kluwer, Dordrecht, 1992). MR 92i:54022

[3] A.V. Arhangel'skiǐ, $C_{p}$-theory, in: M. Hušek and J. van Mill, eds., Recent Progress in General Topology (Elsevier Science Publishers B.V., 1992), 1-56. CMP 93:15

[4] A.V. Arhangel'skiǐ, Embeddings in $C_{p}$-spaces, Topology Appl. 85 (1998), 9-33. MR 99c:54018

[5] J. Baars, Function spaces on first countable paracompact spaces, Bull. Pol. Acad. Sci. 42, 1 (1994), 29-35.

[6] M.M. Choban, General theorems on functional equivalence of topological spaces, Topol. Appl. 89 (1998), 223-239. CMP 99:01

[7] O.G. Okunev, Weak topology of an associated space, and t-equivalence, Math. Notes 46 (1-2) (1990), 334-338. MR 91h:46008

[8] O.G. Okunev, Homeomorphisms of function spaces and hereditary cardinal invariants, Topology Appl. 80 (1997), 177-188. MR 98i:54006

[9] E.G. Pytkeev, Tightness of spaces of continuous functions, Uspekhi Mat. Nauk 37, N 1 (1982), 157-158. (In Russian). MR 83c:54017

[10] V.V. Tkachuk, Some non-multiplicative properties are l-invariant, Comment. Math. Univ. Carolinae 38, N 1 (1997), 169-175. MR 98h:54010

[11] V. Valov, Function spaces, Topol. Appl. 81 (1997), 1-22. MR 98j:54030

[12] N.V. Velichko, The Lindelöf property is l-invariant, Topol. Appl. 89 (1998), 277-283. MR 99h:54025

Département de Mathématiques, Université de Rouen, CNRS UPRES-A 6085, 76821 Mont Saint-Aignan, France

E-mail address: Ahmed.Bouziad@univ-rouen.fr 